\title{
Multiple Arteries in the Renal Transplant
}

\author{
Gioacchino Li Cavoli ${ }^{*}$, Saverio Matranga ${ }^{2}$, Andrea Macaluso ${ }^{2}$, Antonio Amato ${ }^{1}$, Vitalba \\ Azzolina $^{1}$ and Angelo Tralongo ${ }^{1}$
}

\author{
${ }^{1}$ Nephrology Dialysis Renal Transplant Department, Civico Hospital, Palermo Italy \\ ${ }^{2}$ Renal Transplant Surgery Department, Civico Hospital, Palermo Italy
}

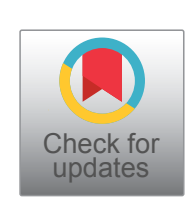

In renal vascular anatomy, compared to the regular presence of the main renal artery, one or more additional renal arteries unilaterally or bilaterally are the commonest arterial anatomical variation of the kidneys. The influence of multiple renal arteries (MRA) graft on the vascular transplant and the clinical outcome is not well established. Contradictory results have been reported if the use of kidney allografts with MRA adversely affects post-transplant graft and patient outcome or increases the incidence of vascular and urologic complications compared with the use of grafts with a single renal artery (SRA). In 2007 Gawish and coll., reported their experience in Kuwait in 35 renal transplants with MRA; they concluded that although transplantation of MRA grafts might carry a relatively higher risk for complications, it is justified because it gives results comparable with those achieved in SRA [1]. Ghods, among 689 renal transplants in Iran, found the presence of MRA as predictor of delayed graft function [2]. Bessede, reviewing 3189 renal transplants performed in France in the period 1971-2010, identifies the presence of MRA graft as risk factor of arterial thrombosis [3]. Ashraf and coll., in 33 live-related kidney transplants with MRA performed from 2006 to 2010 in Pakistan, didn't find significant difference in the warm ischemia with 72 grafts with SRA; they concluded that kidney transplantation using grafts with MRA is equally safe as using grafts with SRA regarding vascular, urological complications, and patient and graft survival [4]. In the metanalysis of Zorgdrager and coll., analyzing a total of 18,289 patients, the recipients of an MRA graft showed a significantly higher incidence of vascular complications compared to those who received an SRA graft, $10.8 \%$ vs. $8.1 \%$, respectively; the incidence of urological complications was also higher in patients who received an MRA graft, 5.5\% vs. $5 \%$ in SRA grafts; no significant differences were observed in the 5-year graft survival and 1- and 5-year patient survival rates. The Authors concluded that MRA grafts were associated with a higher risk of complication and delayed graft function but had comparable long-term outcomes for graft and patient survival [5]. Fitzpatrick and coll., among 106 renal transplants with MRA in the period 2004-2014 in UK, report comparable complication rates with SRA grafts. They conclude that the use of MRA grafts procured by total laparoscopic live donor nephrectomy has comparable complication rates to SRA grafts and should not preclude selection for renal transplantation [6]. In the experience of Sevmis and coll., (199 renal transplant in Turkey 2016-2017), they report that grafts with MRA and grafts with SRA have comparable results in the first post-transplant year [7]. Multiple renal arteries present a technical challenge. Various techniques for arterial reconstruction in cases of MRA grafts were reported. In the bench reconstruction of MRA, it should be used the technique that best fits a particular situation and that the individual transplant surgeon feels most comfortable. In our experience (Figure 1 and Figure 2) the presence of MRA does not increase the risk of vascular or urological complications. According to current literature, we believe that renal transplant with MRA grafts is a safe procedure with no difference in long term outcomes with SRA grafts.

\section{Conflict of Interest}

The Authors state that no conflict of interest exists.

\section{Funding}

None.

\section{Authors Declaration}

The Authors declare that this Article follows the Ethical Guidelines describing animal or human studies. The Authors declare that they have obtained the approval from the interested subject and their institutional review board.

Gioacchino Li Cavoli contributed with the conceptualization of the idea and drafted the article. Saverio Matranga performed the data analysis. All authors participated in editing the article.

*Corresponding author: Gioacchino Li Cavoli, Nephrology Dialysis Renal Transplant Department, Civico Hospital, Francesco Cilea 43, Palermo 90144, Italy, Tel: +393332318100 Accepted: January 29, 2022

Published online: January 31, 2022

Citation: Cavoli GL, Matranga S, Macaluso A, et al. (2022) Multiple Arteries in the Renal Transplant. J Transplant Surg 5(1):87-88

Copyright: (C) 2022 Cavoli GL, et al. This is an open-access article distributed under the terms of the Creative Commons Attribution License, which permits unrestricted use, distribution, and reproduction in any medium, provided the original author and source are credited. 


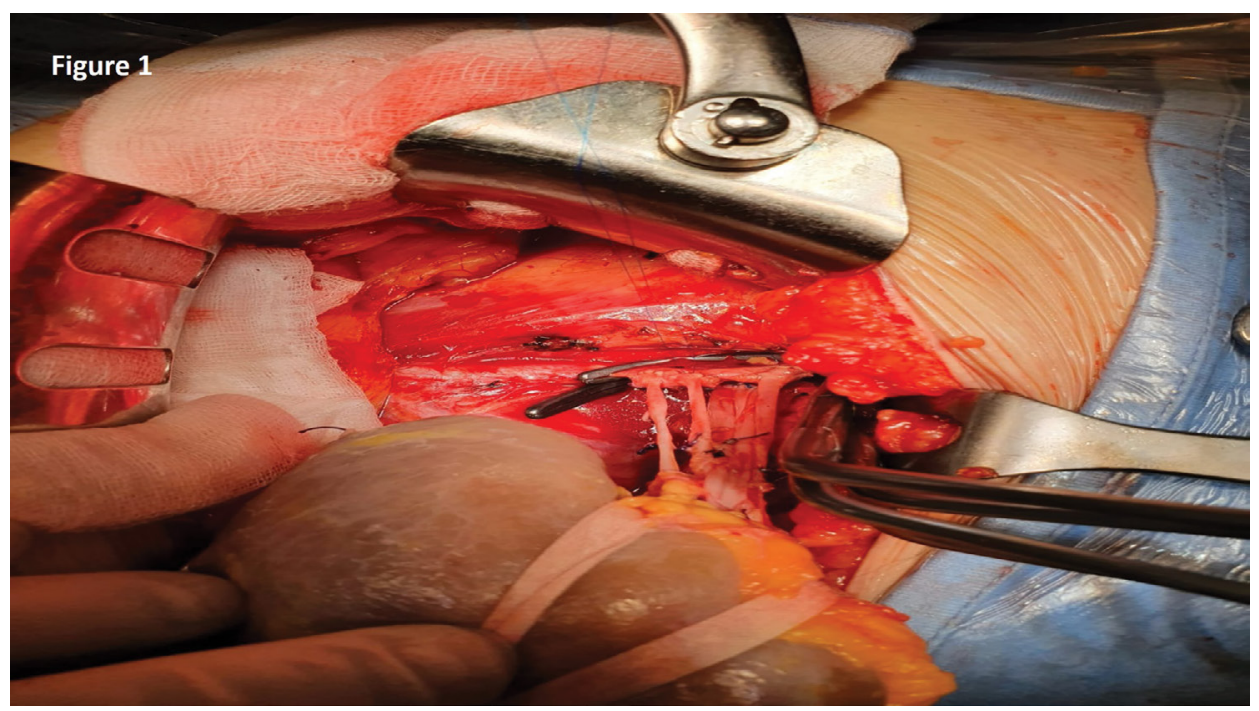

Figure 1: Renal Transplant from deceased donor, with 3 multiple renal arteries (main artery and 2 smaller ones) supplying the graft.

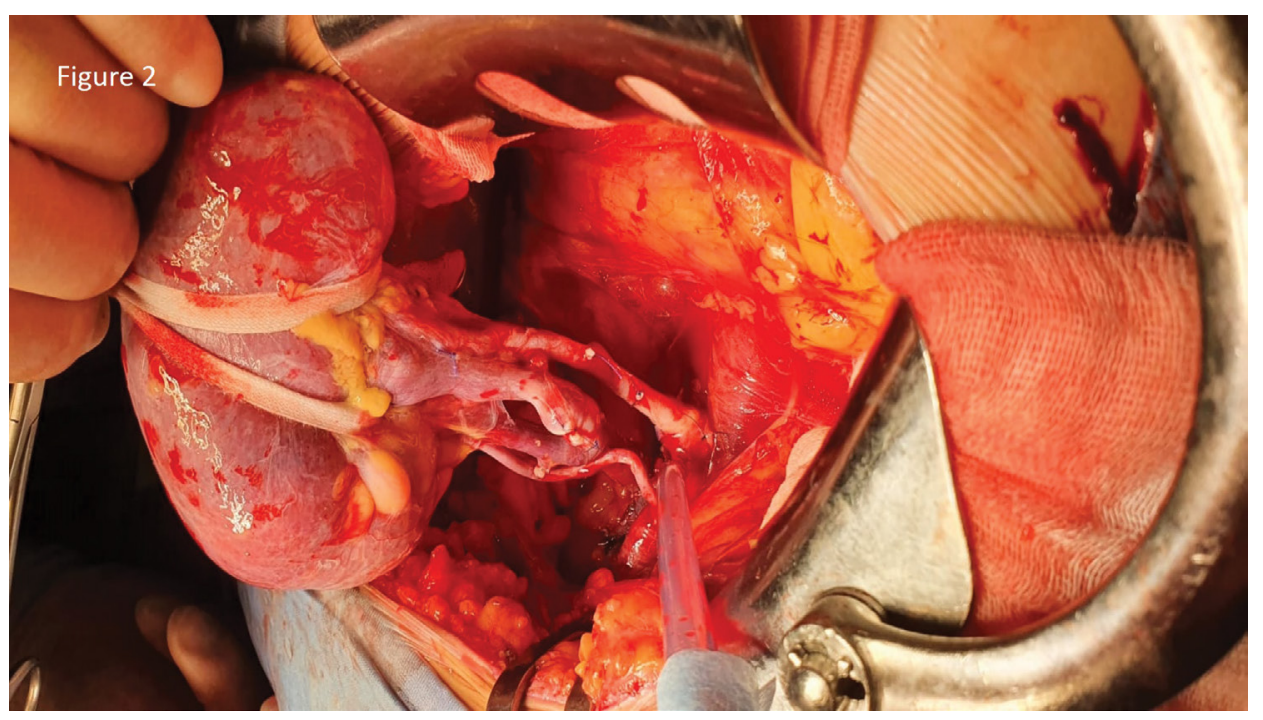

Figure 2: Renal Transplant from deceased donor, with 1 main artery and 1 smaller artery supplying the graft.

\section{References}

1. Gawish AE, Donia F, Samhan M, et al. (2007) Outcome of renal allografts with multiple arteries. Transplant Proc 39: 1116-1117.

2. Ghods AJ, Savaj S, Abbasi M, et al. (2007) The incidence and risk factors of delayed graft function in 689 consecutive living unrelated donor renal transplantation. Transplant Proc 39: 846847.

3. Bessede T, Droupy S, Hammoudi Y, et al. (2012) Surgical prevention and management of vascular complications of kidney transplantation. Transpl Int 25: 994-1001.
4. Ashraf HS, Hussain I, Siddiqui AA, et al. (2013) The outcome of living related kidney transplantation with multiple renal arteries. Saudi J Kidney Dis Transpl 24: 615-619.

5. Zorgdrager M, Krikke C, Hofker SH, et al. (2016) Multiple renal arteries in kidney transplantation: A Systematic review and meta-analysis. Ann Transplant 21: 469-478.

6. Fitzpatrick J, Chmelo J, Nambiar A, et al. (2020) Recipient outcomes in total laparoscopic live donor nephrectomy with multiple renal vessels. Urol Ann 12: 266-270.

7. Sevmis M, Demir ME, Merhametsiz O, et al. (2021) Grafts with multiple renal arteries in kidney transplantation. Transplant Proc 53: 933-940.

DOI: $10.36959 / 338 / 339$ 